\title{
Schlafstörungen
}

\section{Verbesserte Schlafqualität}

Klinische Studien zeigen, dass retardiertes Melatonin bei Alzheimerpatienten positiv auf die kognitive Leistungsfähigkeit und die Schlafqualität wirkt.

Toxische Beta-Amyloid-Plaques, die sich vermehrt im Hirngewebe ansammeln, spielen eine Schlüsselrolle bei der Entstehung der Alzheimerdemenz. Offenbar gehen die schädlichen Proteinablagerungen bei älteren Menschen zunehmend auch mit Schlafstörungen einher. $\mathrm{Zu}$ wenig Schlaf könnte also auch die Konzentration des Alzheimerpeptids im Gehirn erhöhen und so eine Demenz begünstigen. Ein mangelhafter Abbau durch gestörten Schlaf kann demnach zur Akkumulation toxischer Produkte führen, die die Entzündungen verstärken. Durch eine Verbesserung des Schlafs könnte auch die vielfache Problematik der Alzheimer-Demenz sowohl für Patienten als auch für Angehörige bzw. Betreuer erleichtert werden. Etwa ein Drittel der erwachsenen Menschen leidet unter Schlafstörungen, wobei die Beschwerden mit steigendem Alter zunehmen.

\section{Insomnien beeinträchtigen Körper, Geist und Seele}

Vor allem älteren Menschen werden bei Angst- und Schlafstörungen Benzodiazepine verordnet - und nicht selten werden diese auch länger als empfohlen eingenommen. Bei der herkömmlichen Schlafmedikation bestehen Bedenken hinsichtlich eines Insomnie-Rebounds und/oder von Entzugserscheinungen. Die Anwendung bei Menschen mit Demenz ist mit Vorsicht geboten - allein wegen des erhöhten Risikos für Stürze, des Abhängigkeitspotential und der Gefahr des Delirs.

\section{Retardiertes Melatonin}

Circadin $^{\circledR}$, eine 2 mg-Melatonin-Retardtablette ist eine innovative Behandlung der primären Insomnie bei Patienten $>55$ Jahre mit durch schlechte Schlafqualität gekennzeichneten Schlafproblemen. Es hat eine unterschiedliche Wirkweise zu Benzodiazepinen und Benzodiazepin-ähnlichen Medikamenten und ist das erste Medikament aus der neuen Klasse der Melatonin-Rezeptor-Agonisten. Nur durch die Melatonin-Retard-Formulierung ist eine gleichmäßige Wirkung über die ganze Nacht gewährleistet. Eine gezielte Zufuhr von langwirksamen Melatonin ist in der Lage, den physiologische Wach-Schlaf-Rhythmus wieder herzustellen und damit die Schlafqualität wesentlich zu verbessern.

Weitere Ergebnisse einer multizentrischen Observationsstudie zeigen für retardiertes Melatonin

- eine Verbesserung der Schlafqualität bei $82 \%$ der Patienten

- bei $76 \%$ der Patienten eine deutliche Steigerung der Munterkeit am Morgen

- die anhaltende Wirkung auch nach Therapieende

- keine Rebound-Insomnie oder Entzugssymptome

Nahezu $80 \%$ der Patienten konnten durch Circadin $^{\circledast}$ die Einnahme von Hypnotika beenden [1].

Der endogene Melatoninspiegel sinkt bereits bei vorklinischen Stadien von Alzheimer. Da Melatonin eine wichtige Rolle für die Schlafqualität spielt und eine schlechte Schlafqualität mit Alzheimer in Zusammenhang gebracht wird, gilt es die Wirkung der Gabe des fehlenden Hormons auf Alzheimerpatienten und den Zusammenhang mit einem besseren Schlaf zu erforschen.

Die Ergebnisse der explorativen randomisierten, placebokontrollierten Studie von Wade et al zeigen über einen Zeitraum von 6 Monaten die Sicherheit und Wirksamkeit der Gabe von retardiertem Melatonin als Begleittherapie zur Standardmedikation für Alzheimerpatienten. Die Studie belegt eine positive Wirkung auf die kognitive Leistungsfähigkeit, die Schlafqualität und das Durchschlafvermögen bei den teilnehmenden Alzheimerpatienten [2].

\section{Literatur}

1. Hajak G et al (2015) Lasting treatment effects in a postmarketing surveillance study of prolonged-release melatonin. Int Clin Psychopharmacol 30(1):36-42

2. Wade AG et al (2014) Add-on prolonged-release melatonin for cognitive function and sleep in mild to moderate Alzheimer's disease: a 6-month, randomized, placebo-controlled, multicenter trial. Clin Interv. Aging 18(9):947-961

psychopraxis. neuropraxis 2016 $\cdot 19: 95$

DOI 10.1007/s00739-016-0326-x

Online publiziert: 27. April 2016

C) Springer-Verlag Wien 2016

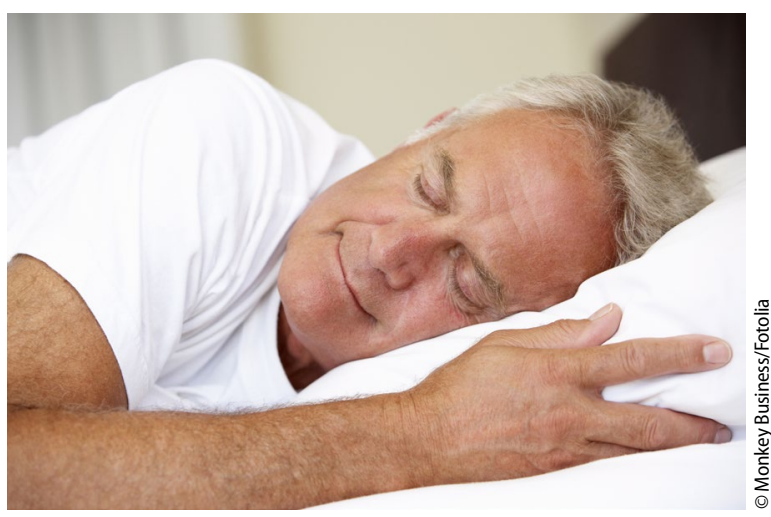

\ Erholsamer Schlaf unterstützt Körper, Geist und Psyche 